\title{
The role of radiosurgery for hemangiopericytomas
}

\author{
Steven D. Chang, M.D., and Gordon T. Sakamoto, B.A. \\ Department of Neurosurgery, Stanford University School of Medicine, Stanford, California
}

\begin{abstract}
Object. Hemangiopericytomas represent a small subset of meningeal tumors. Despite their relatively uncommon nature, they are aggressive tumors known for recurrence. Resection is the standard treatment in most, although regrowth and metastases are common even after resection. The authors evaluate the role of stereotactic radiosurgery in the treatment of recurrent hemangiopericytomas.

Methods. In a review of the Stanford radiosurgery patient database between 1989 and 2002, the authors found eight patients with recurrent hemangiopericytoma who underwent stereotactic radiosurgery. The mean age of this population was 45.1 years (range 24-67 years). All patients had been previously treated with resection, and five patients $(63 \%)$ had undergone conventional radiotherapy. The mean radiosurgery dose to the tumor margin was 20.5 Gy (range 16-24 Gy). The mean clinical and radiographic follow-up period was 44 months (range 8-77 months). Of the eight tumors treated with radiosurgery, six decreased in size and two ultimately progressed. There were no radiosurgeryrelated complications.

Conclusions. Stereotactic radiosurgery of hemangiopericytomas can result in increased tumor control and should be considered as a treatment option for patients in whom the diagnosis has been established and in whom residual tumor is demonstrated postoperatively. Close clinical and radiographic follow-up evaluation is necessary in this patient population because of the high rate of local recurrence and distant metastases.
\end{abstract}

KEY WORDS • hemangiopericytoma • radiosurgery • stereotaxy

Hemangiopericytomas of the central nervous system are highly vascular, rapidly growing lesions of the meninges. They represent a small minority of brain tumors; their radiographic appearance and clinical presentation often result in their being diagnosed as meningiomas..$^{10,12,20,23}$ Some authors have considered this tumor type to be similar to an angioblastic meningioma, but the authors of more recent evaluations have classified this tumor as a distinct group from meningiomas. The standard treatment for hemangiopericytomas is resection whenever possible. ${ }^{26}$ As with benign meningiomas, however, total excision often cannot be achieved when hemangiopericytomas arise from the skull base or involve the venous sinuses. Furthermore, even after gross-total resection, recurrence is common. Conventional radiotherapy has been proposed for postsurgical treatment of hemangiopericytomas, even when a gross-total resection has been achieved, because of the high rate of recurrence. . $^{2,615,21,28,29,32}$ The focal, welldefined nature of these lesions on MR imaging, however, makes them attractive targets for stereotactic radiosurgery, and radiosurgery has been shown to be an effective treatment for recurrent hemangiopericytomas. ${ }^{4,16,22,26}$ We report our experience using radiosurgery to treat eight patients with postresection-recurrent or residual hemangiopericytomas.

Abbreviations used in this paper: $\mathrm{CT}=$ computerized tomography; LINAC = linear accelerator; $\mathrm{MR}=$ magnetic resonance.

\section{CLINICAL MATERIAL AND METHODS}

\section{Patient Population}

Between 1992 and 2002, eight patients with nine hemangiopericytomas were treated with stereotactic radiosurgery. Their mean age was 45.1 years (range 24-67 years). There were six men and two woman (Table 1). Six patients $(75 \%)$ exhibited symptoms at the time of treatment whereas two asymptomatic patients were treated after serial MR imaging revealed hemangiopericytoma progression. All tumors were residual or recurrent lesions demonstrated after prior resection, in which pathological examination confirmed the diagnosis. Three patients had undergone two or more previous resections, whereas five patients had undergone one prior operation. Five patients (63\%) had previously received cranial irradiation (mean dose $51 \mathrm{~Gy}$, range $45-56 \mathrm{~Gy}$ ). Clinical symptoms prior to treatment varied but correlated with the lesion's location. Presenting symptoms included cranial nerve deficits in four cases, headaches in three, seizures in one, and motor or sensory deficits in one.

\section{Treatment and Follow-Up Evaluation}

Four patients were treated with LINAC-based stereotactic radiosurgery that involved a technique modeled after that of Winston and Lutz. ${ }^{33}$ Localization was performed using CT images fused to 2-mm slice contrastenhanced MR images. Multiple noncoplanar arc radiation 
TABLE 1

Summary of demographic and treatment-related data

\begin{tabular}{lc}
\hline \multicolumn{1}{c}{ Characteristic } & Value \\
\hline age (years) & \\
mean & 45.1 \\
range & $24-67$ \\
male/remale & $6: 2$ \\
patients w/ prior op & 8 \\
one craniotomy & 5 \\
two craniotomies & 2 \\
three craniotomies & 1 \\
patients with prior radiotherapy & 5 \\
radiotherapy dose (Gy) & \\
mean & 51 \\
range & $45-56$ \\
follow-up period (mos) & \\
mean & 44 \\
range & $8-77$ \\
\hline
\end{tabular}

(from either a 4 or $6 \mathrm{mV}$ LINAC) was used, with an $80-\mathrm{cm}$ source to the axis distance. Circular secondary collimators ranged in diameter from 7.5 to $25 \mathrm{~mm}$ (mean 12.8 $\mathrm{mm}$ ). The treatment dose was selected on the basis of tumor size, location, proximity of critical neurological structures, number of isocenters (tumors with complex geometric shapes were treated with multiple isocenters), and any history of conventional radiotherapy. The treatment dose ranged from 16 to 24 Gy (mean 20 Gy), which was prescribed to the $80 \%$ isodose contour at the edge of tumor (Table 2).

In four patients the CyberKnife (Accuray, Inc., Sunnyvale, CA) was used. Patients were initially placed on the treatment couch, and a thermoplastic mask was created and attached to the couch. Patients were then transferred to a CT scanner (Lightspeed; General Electric, Milwaukee, WI), and $125 \mathrm{ml}$ of Omnipaque contrast was administered. A series of CT scans was acquired at $1.25-\mathrm{mm}$ slice thickness. The scans were then transferred to the treatment planning computer via a standard DICOM network. The tumor volume was outlined, and inverse treatment planning was then performed to achieve a highly conformal treatment plan, with attempts to minimize the dose to critical structures. The treatment dose to tumor periphery was $18 \mathrm{~Gy}$ in one patient, $22 \mathrm{~Gy}$ in two patients, and $24 \mathrm{~Gy}$ in the remaining patient.

Radiographic follow-up evaluation included 2- to 5$\mathrm{mm}$ slice thickness Gd-enhanced MR images obtained every 4 months for the 1 st year after treatment, every 6 months for the 2nd year, and annually thereafter. Clinical follow-up examination was conducted at the same intervals. The mean clinical and radiographic follow-up period was 44 months (range 8-77 months).

\section{RESULTS}

\section{Neuroimaging-based Outcome}

A decrease in tumor size was observed in six patients (75\%) (Fig. 1), the lesion disappeared in one (12.5\%), and it progressed in one patient $(12.5 \%)$. In the case in which MR imaging-based tumor disappearance was demonstrat-
TABLE 2

Summary of treatment parameters

\begin{tabular}{ll}
\hline \multicolumn{1}{c}{ Parameter } & Value \\
\hline treatment modality (no. of cases) & \\
isocentric & 4 \\
nonisocentric & 4 \\
dose to tumor margin $(\mathrm{Gy})$ & 20.8 \\
mean & $16-24$ \\
range & \\
maximal dose $(\mathrm{Gy})$ & 27.5 \\
mean & $22-31$ \\
range & \\
treatment isodose line $(\%)$ & 77 \\
mean & $74-81$ \\
range &
\end{tabular}

ed 12 months posttreatment, recurrence was found after an additional 12 months and required another resection. This same patient was also noted to have metastases of his tentorial hemangiopericytoma to the temporalis muscle on the ipsilateral side (treated with resection). No other distant metastases were noted in this population. There was no radiological evidence of edema or necrosis in the tissue adjacent to the tumor in any patient in this series.

\section{Clinical Outcome}

Two patients (25\%) in the series reported clinical improvement, both involving resolution or reduction in headaches, six patients $(75 \%)$ indicated no change in symptoms, and no patient described a worsening of clinical status. Of the patients with cranial nerve deficits, none experienced any improvement in these deficits. The one patient in whom there was MR imaging evidence of tumor progression did not develop new clinical symptoms but continued to suffer baseline cranial nerve deficits. The patient with the hemangiopericytoma metastasis to the ipsilateral temporalis muscle noted the development of a mass under the temporal scalp, but experienced no neurological deficits.

The patient with documented tumor progression despite radiosurgery died during the follow-up period of complications related to a subsequent open surgery performed to excise the hemangiopericytoma. The overall 3-year survival rate was $88 \%$.

\section{DISCUSSION}

Meningeal hemangiopericytomas often have clinical and radiographic presentations similar to meningiomas. ${ }^{10,12,20}$ Hemangiopericytomas, however, are much more aggressive lesions, associated with high rates of local or distant recurrences, ${ }^{1-3,5,10-12,17,18,20,23,27,31}$ and thus can behave similarly to other malignant lesions. ${ }^{24}$ Resection is the initial treatment of choice, ${ }^{26}$ but the highly vascular nature of the lesion and the location of some along the skull base results in mortality rates between 9 and $24 \%{ }^{12,14,23,25}$ Furthermore, the median time from resection to recurrence is 12 months, ${ }^{8}$ and distant metastases have been noted to appear between a mean of 63 and 99 months after initial diagnosis. $7,12,14,23,25$

Because of the recurrent nature of these lesions, exter- 

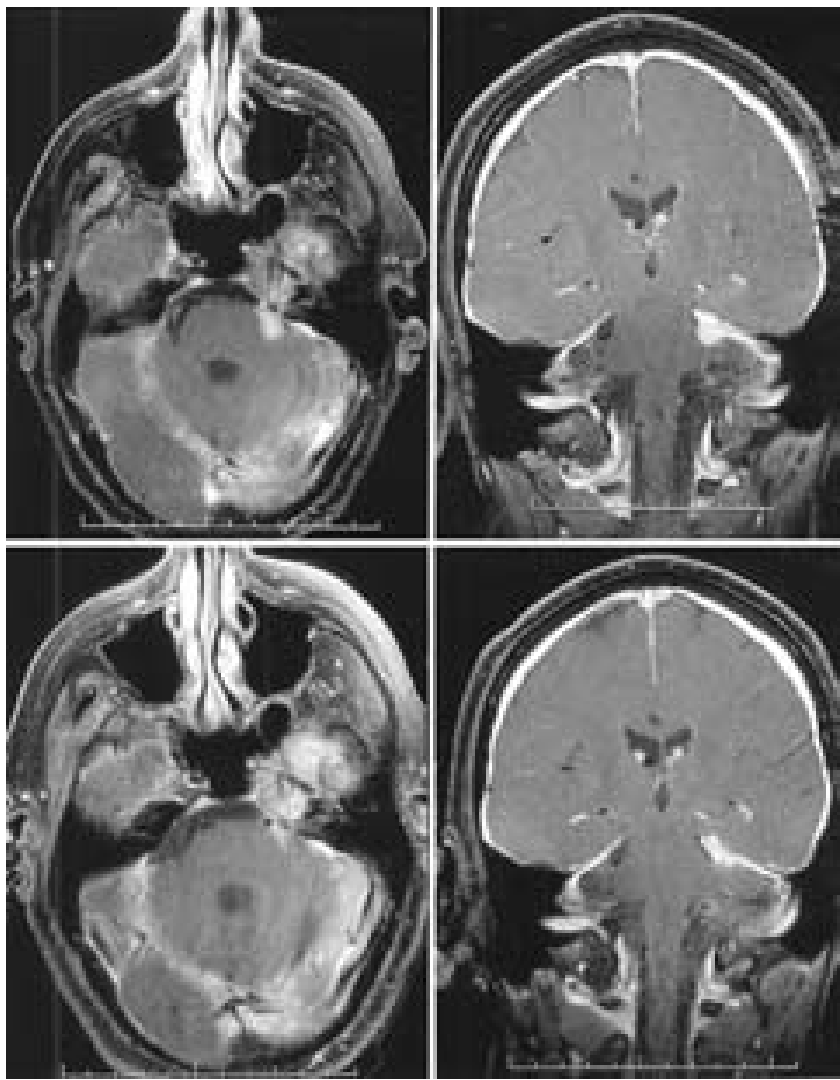

Fig. 1. Magnetic resonance images obtained in a 54-year-old man who presented with a left cavernous sinus hemangiopericytoma extending along the tentorial edge. After the patient underwent resection and conventional external-beam radiotherapy, a residual contrast-enhancing nodule was noted along the left tentorial edge in both axial (upper left) and coronal (upper right) Gdenhanced MR images. The patient underwent stereotactic radiosurgery (22-Gy dose), and at 11-month follow-up examination the tumor was demonstrated to be smaller on axial (lower left) and sagittal (lower right) MR images.

nal-beam radiotherapy has been used in the postoperative setting. Dube and Paulson ${ }^{6}$ reported one of the first radiotherapy-based treatments of a hemangiopericytoma, noting a complete response. Mira, et al., ${ }^{21}$ reported on a series of 11 patients in whom hemangiopericytomas were treated with more than 29 courses of radiotherapy, noting clinical response in 26 of the 29 courses, and complete regression following 14 courses. The authors of subsequent reports have also documented the benefit of radiotherapy in patients previously treated with surgery, even when a gross-total resection had been achieved. $5,7-9,12,13,15,19,32$ Dufour, et al., ${ }^{7}$ demonstrated that postoperative radiotherapy decreased the local recurrence rate to $12.5 \%$ after 50 to 64-Gy treatments compared with $88 \%$ after surgery alone. Guthrie, et al., ${ }^{12}$ reported that radiotherapy after the first operation extended the mean time before first recurrence from 34 to 75 months and extended survival from 62 to 92 months. Glaholm, et al., ${ }^{9}$ noted that even in those patients who had undergone resection previously, radiotherapy alone resulted in improved neurological performance in $38 \%$ of patients with inoperable hemangioperi- cytomas. Uemura, et al. ${ }^{32}$ documented that radiotherapy even provided a benefit if delivered prior to resection, making the tumors less vascular and allowing resection with minimal blood loss.

\section{Rationale for Radiosurgery}

Based on the aforementioned data, as well as on their well-defined nature, hemangiopericytomas represent reasonable radiosurgical targets; some authors have even asserted that the highly vascular nature of these tumors likely increases their favorable response to treatment. ${ }^{4}$ The primary challenge with resection alone is the a high rate of postoperative recurrence. Multiple resections are feasible, although not attractive because of the appreciable morbidity associated with multiple operations. In radiosurgery the efficacy of resection is combined with the more minimal rate of radiotherapy-induced morbidity.

Stereotactic radiosurgery should be particularly beneficial for skull base hemangiopericytomas, because these tumors usually present as reasonably small as a result of their proximity to cranial nerves, whose dysfunction often heralds the presence of tumor. Although conventional radiotherapy of skull base hemangiopericytomas may include critical skull base structures, such as the pituitary, optic nerves, and brainstem, the steep dose gradient that can be achieved using radiosurgery minimizes the radiation delivered to these areas. Consequently, it is possible to deliver a much larger, and presumably more efficacious, dose to the tumor without exceeding the radiation tolerance of normal tissues.

\section{Previous Radiosurgical Reports}

The first preliminary stereotactic radiosurgery report for hemangiopericytomas was reported by Coffey, et al. ${ }^{4}$ A total of 11 hemangiopericytomas were treated in five patients, all of whom had undergone at least one prior craniotomy and three of whom had undergone prior radiotherapy to doses of 50 to $53 \mathrm{~Gy}$. Marginal doses of 12 to $18 \mathrm{~Gy}$ were used, and the mean follow-up period was 14.8 months. Of the nine treated tumors for which radiographic follow-up data were available, eight decreased in size and one was no longer visible. Galanis, et al., ${ }^{8}$ reported on 10 patients (five of whom were also included in the series by Coffey, et al.) in whom 20 hemangiopericytomas were treated with stereotactic radiosurgery (doses of 12-18 Gy). Seven of the 10 patients had previously undergone radiotherapy (dose range 3060-6400 cGy, median 5580 cGy). Fourteen of the hemangiopericytomas decreased in size, four disappeared radiographically, and two were stable in size.

Payne, et al., ${ }^{22}$ analyzed data obtained in 12 patients with 15 stereotactic radiosurgery-treated intracranial hemangiopericytomas and reported follow-up data in 10 of these patients. All but one of the patients had undergone prior craniotomies (mean number of surgeries 2.9), and four patients had undergone prior fractionated radiotherapy. The mean peripheral dose was 14 Gy (range 2.8-25 Gy), and the mean clinical follow-up period was 24.8 months during which nine tumors decreased in size and three additional tumors were stable in size. Four of the nine tumors that decreased in size, however, subsequently 
had increased in size after a mean of 22 months postradiosurgery.

In a recent report by Sheehan, et al., ${ }^{26}$ the authors reviewed a series of 14 patients with 15 hemangiopericytomas treated with radiosurgery. Twenty-seven prior craniotomies had been conducted in this population, and seven patients had previously undergone radiotherapy (dose range 30-61 Gy, mean $47.3 \mathrm{~Gy}$ ). The marginal radiosurgery doses ranged from 11 to $20 \mathrm{~Gy}$, and the follow-up period ranged from 5 to 76 months (mean 31.3 months). On follow-up imaging, tumor regression was demonstrated in 12 of 15 lesions; three tumors were observed to have increased in size. Despite this $80 \%$ local control rate, $29 \%$ of the patients developed remote metastases, indicating that radiosurgery provided no protection from distant metastatic spread.

\section{The Stanford Series}

The findings in the present series confirm those of earlier reports. Tumor control was noted in $75 \%$ of the hemangiopericytomas treated during a mean 44 months follow-up period; this is comparable to the control rates documented in other series., ${ }^{4,822,26}$ Dose rates to tumor periphery in this series were slightly higher (mean 20.8 Gy) compared with those in other series (mean dose range 14-16 Gy)., ${ }^{4,22,26}$ This higher mean dose, however, did not translate to increased tumor control rates or radiosurgeryrelated complication rates, perhaps due to the relatively small number of patients in all the reported series including the present one. As in the other series, all of our patients had previously undergone open resection, with some patients having undergone multiple excisions. In this population, further resection can be associated with increasing morbidity rates, a fact that makes radiosurgical treatment attractive.

Two additional observations confirm those made in other series. First, radiosurgery is a focal treatment and does not necessarily prevent regional and distant metastases. Metastases outside the treatment area were noted in this and other series, often developing within only a few years of treatment ${ }^{4,8,22,26}$ or even decades after initial treatment. ${ }^{30}$ Second, the aggressive nature of hemangiopericytomas can result in initial decreases in tumor size or even disappearance, only to be followed by later regrowth. This was observed in the present study and also noted previously. ${ }^{22}$ Both of these issues support the need for close clinical and radiographic follow-up observation in this patient population.

\section{CONCLUSIONS}

Resection remains the initial treatment for hemangiopericytomas, because histopathological diagnosis is required to distinguish them from meningiomas. Once resection is achieved, however, the high rates of hemangiopericytoma recurrence may be addressed by performing stereotactic radiosurgery, which has shown to provide significant benefit. Radiotherapy has been used to treat hemangiopericytomas, but their well-defined contrast-enhancing targets make them suitable for radiosurgery while minimizing dose to normal tissue. As suggested by this and previous reports, such hemangiopericytoma recur- rences or remnants are readily, and very safely, treated radiosurgically, with increased tumor control rates. Because radiosurgery is a focal treatment, however, it does not eliminate the possibility of regional or distant metastases, which remain sources of significant morbidity and mortality in this patient population.

\section{Acknowledgment}

We thank Beth Hoyte for assistance with production of the figures.

\section{References}

1. Baird M, Gallagher PJ: Recurrent intracranial and spinal meningiomas: clinical and histological features. Clin Neuropathol 8: 41-44, 1989

2. Bastin KT, Mehta MP: Meningeal hemangiopericytoma: defining the role for radiation therapy. J Neurooncol 14:277-287, 1992

3. Boker DK, Stark HJ: The proliferation rate of intracranial tumors as defined by the monoclonal antibody KI 67. Application of the method to paraffin embedded specimens. Neurosurg Rev 11:267-272, 1988

4. Coffey RJ, Cascino TL, Shaw EG: Radiosurgical treatment of recurrent hemangiopericytomas of the meninges: preliminary results. J Neurosurg 78:903-908, 1993

5. de la Monte SM, Flickinger J, Linggood RM: Histopathologic features predicting recurrence of meningiomas following subtotal resection. Am J Surg Pathol 10:836-843, 1986

6. Dube VE, Paulson JF: Metastatic hemangiopericytoma cured by radiotherapy. A case report. J Bone Joint Surg Am 56: 833-835, 1974

7. Dufour H, Metellus P, Fuentes S, et al: Meningeal hemangiopericytoma: a retrospective study of 21 patients with special review of postoperative external radiotherapy. Neurosurgery 48:756-763, 2001

8. Galanis E, Buckner JC, Scheithauer BW, et al: Management of recurrent meningeal hemangiopericytoma. Cancer 82: 1915-1920, 1998

9. Glaholm J, Bloom HJ, Crow JH: The role of radiotherapy in the management of intracranial meningiomas: the Royal Marsden Hospital experience with 186 patients. Int J Radiat Oncol Biol Phys 18:755-761, 1990

10. Goellner JR, Laws ER Jr, Soule EH, et al: Hemangiopericytoma of the meninges. Mayo Clinic experience. Am J Clin Pathol 70:375-380, 1978

11. Gupta PK, Sastry Kolluri VR, Das S, et al: Recurrences in meningioma after surgery. Acta Neurochir 100:104-107, 1989

12. Guthrie BL, Ebersold MJ, Scheithauer BW, et al: Meningeal hemangiopericytoma: histopathological features, treatment, and long-term follow-up of 44 cases. Neurosurgery 25: 514-522, 1989

13. Hashiguchi K, Inamura T, Iwaki T, et al: [Increased intracranial pressure caused by obstruction of torcular herophili with hemangiopericytoma: a case report.] No Shinkei Geka 29: $175-179,2001(\mathrm{Jpn})$

14. Jaaskelainen J, Servo A, Haltia M, et al: Intracranial hemangiopericytoma: radiology, surgery, radiotherapy, and outcome in 21 patients. Surg Neurol 23:227-236, 1985

15. Jha N, McNeese M, Barkley HT Jr, et al: Does radiotherapy have a role in hemangiopericytoma management? Report of 14 new cases and a review of the literature. Int J Radiat Oncol Biol Phys 13:1399-1402, 1987

16. Kocher M, Voges J, Staar S, et al: Linear accelerator radiosurgery for recurrent malignant tumors of the skull base. Am J Clin Oncol 21:18-22, 1998

17. Kulali A, von Wild K, Hobik HP: Subarachnoid haemorrhage 
with acute cauda symptom due to spinal tumor. Neurochirurgia (Stuttg) 32:87-90, 1989

18. Kumar PP, Good RR, Skultety FM, et al: Spinal metastases from pituitary hemangiopericytic meningioma. Am J Clin Oncol 10:422-428, 1987

19. McDonald JV: Meningeal hemangiopericytoma. Neurosurgery 26:176, 1990

20. Mena H, Ribas JL, Pezeshkpour GH, et al: Hemangiopericytoma of the central nervous system: a review of 94 cases. Hum Pathol 22:84-91, 1991

21. Mira JG, Chu FC, Fortner JG: The role of radiotherapy in the management of malignant hemangiopericytoma: report of eleven new cases and review of the literature. Cancer 39: 1254-1259, 1977

22. Payne BR, Prasad D, Steiner M, et al: Gamma surgery for hemangiopericytomas. Acta Neurochir 142:527-537, 2000

23. Pitkethly DT, Hardman JM, Kempe LG, et al: Angioblastic meningiomas; clinicopathologic study of 81 cases. J Neurosurg 32:539-544, 1970

24. Scheithauer BW: Tumors of the meninges: proposed modifications of the World Health Organization classification. Acta Neuropathol 80:343-354, 1990

25. Schroder R, Firsching R, Kochanek S: Hemangiopericytoma of meninges. II. General and clinical data. Zentralbl Neurochir 47:191-199, 1986

26. Sheehan J, Kondziolka D, Flickinger J, et al: Radiosurgery for treatment of recurrent intracranial hemangiopericytomas. Neurosurgery 51:905-911, 2002
27. Skullerud K, Loken AC: The prognosis in meningiomas. Acta Neuropathol 29:337-344, 1974

28. Someya M, Sakata KI, Oouchi A, et al: Four cases of meningeal hemangiopericytoma treated with surgery and radiotherapy. Jpn J Clin Oncol 31:548-552, 2001

29. Staples JJ, Robinson RA, Wen BC, et al: Hemangiopericytoma-the role of radiotherapy. Int J Radiat Oncol Biol Phys 19:445-451, 1990

30. Suzuki H, Haga Y, Oguro K, et al: Intracranial hemangiopericytoma with extracranial metastasis occurring after 22 years. Neurol Med Chir 42:297-300, 2002

31. Tsuji N, Nishiura I, Koyama T: Extradural multiple spinal meningioma. Literature review a case report. Neurochirurgia (Stuttg) 29:124-127, 1986

32. Uemura S, Kuratsu J, Hamada J, et al: Effect of radiation therapy against intracranial hemangiopericytoma. Neurol Med Chir 32:328-332, 1992

33. Winston KR, Lutz W: Linear accelerator as a neurosurgical tool for stereotactic radiosurgery. Neurosurgery 22:454-464, 1988

Manuscript received March 14, 2003.

Accepted in final form April 14, 2003.

Address reprint requests to: Steven D. Chang, M.D., Department of Neurosurgery, Stanford University School of Medicine, 300 Pasteur Drive, Room R-225, Stanford, California 94305. email: sdchang@stanford.edu. 